\title{
D’un Bercy à l’autre, les états de l'État
}

\author{
Entretien avec Maryvonne Le Brignonen, Directeur de Tracfin réalisé par Marie \\ Alauzen et Fabien Gélédan
}

Maryvonne Le Brignonen est inspectrice des finances, elle peut témoigner à 45 ans d'un riche parcours dans la haute fonction publique, qui l'a menée de l'Inspection générale des finances, à la Direction générale des finances publiques (DGFiP), où elle a conduit le projet de prélèvement à la source de l'impôt sur le revenu, avant d'être nommée à la direction de Tracfin (voir le tableau 1 ci-dessous). C'est dans cette partie de sa carrière, de sa sortie de l'ÉNA, en 2010, à aujourd'hui qu'elle a accepté de se replonger, pour évoquer les différents états de l'État qu'elle a été amenée à expérimenter'

Tableau 1 : Présentation des institutions, dans l'ordre d'évocation

L'Inspection générale des finances est l'un des corps d'inspection de l'État. Rattachée aux ministères économiques et financiers, elle exerce une mission de contrôle et d'audit interne de l'administration, d'étude, de conseil et d'évaluation, pour le compte du ministre chargé de l'Économie et du Budget, et peut, le cas échéant recevoir des missions d'autres administrations publiques nationales et internationales. La diversité des missions des inspecteurs est soulignée par Maryvonne Le Brignonen au cours de l'entretien.

L'activité de l'Inspection, souvent décrite comme intense, est l'objet d'une faible publicité. La publication des travaux se fait au cas par cas, généralement à la discrétion de l'instance commanditaire.

La Direction générale des finances publiques est l'une des directions du ministère de l'Économie, des Comptes publics et de la Relance. Constituée par la

${ }^{1}$ L'entretien aurait dû avoir lieu dans les locaux de Tracfin, situés à Montreuil, en bordure de Paris. Mais, depuis le deuxième confinement qui avait pris effet le 29 octobre 2020 à minuit, les consignes sanitaires étaient de privilégier le travail à distance. C'est donc à travers à une application de visioconférence que nous nous rencontrons, le 16 novembre, par un après-midi gris et venteux. En dépit des perturbations liées à l'application recommandée par Tracfin pour ses niveaux de sécurité et testée quelques jours auparavant, la discussion pour laquelle nous avions envoyé une grille de questions plusieurs semaines à l'avance a duré près de 2 heures. Maryvonne Le Brignonen a revu la transcription pour supprimer les redondances et marques d'oralité. L'appareil de notes de bas de page précise des références documentaires et explicite les éléments de contexte pourraient échapper à la compréhension du lecteur. 
fusion, en 2008 de la Direction générale des impôts et la Direction générale de la comptabilité publique, elle se situe, en effectifs au deuxième rang des organisations civiles de l'État. Elle gère la fiscalité de l'État et des collectivités territoriales, définit les règles juridiques, assure le recouvrement et instruit le contentieux fiscal. Appuyée sur un dense réseau de services déconcentrés, elle est responsable de l'ensemble des domaines juridiques et techniques ayant trait au recouvrement de l'impôt des particuliers et des entreprises. À ce titre, elle maintient l'un des liens les plus anciens de la relation entre l'État et les citoyens.

Pionnière de l'administration électronique, la DGFiP a conçu puis continuellement perfectionné le service de déclaration des revenus en ligne, avant de mettre en place, la retenue à la source de l'impôt sur le revenu des particuliers, longuement évoquée dans le présent entretien, notamment parce qu'elle a réactivé un débat révolutionnaire.

Tracfin est un service de renseignement membre du premier cercle du renseignement français, moins connu du grand public que ses homologues de la Direction générale de la sécurité extérieure (DGSE), Direction générale de la sécurité intérieure (DGSI). Il concourt à la lutte contre les circuits financiers clandestins, le blanchiment d'argent, le financement du terrorisme et la défense des intérêts fondamentaux de la nation. Placé sous l'autorité du ministère de l'Économie, des Finances et de la Relance, le service est chargé de recueillir, d'analyser et d'enrichir les « déclarations de soupçon » que les «professionnels assujettis » c'est-à-dire un certain nombre de professions définies par le Code monétaire et financier (banque, assurance, changeurs, notaires, avocats, marchands d'art, agents immobiliers, etc.) sont tenus de lui déclarer lorsqu'ils repèrent des phénomènes irréguliers.

À la fois discret et donc difficile d'accès en dehors des sites web et de quelques apparitions publiques, il est en même temps partiellement public et lisible pour ce petit nombre de professions. Cette double nature de Tracfin en fait un espace heuristique pour examiner un mode de présence travaillé pour être diaphane.

Pouvez-vous retracer sommairement votre parcours dans la haute fonction publique, de l'École nationale d'administration à Tracfin?

J'ai un parcours particulier — il l'est de moins en moins aujourd'hui, mais le demeurait à l'époque - dans la haute fonction publique. J'ai d'abord fait carrière dans le secteur privé, durant une petite dizaine d'années dans le domaine de l'audit et du commissariat aux comptes, au sein de plusieurs cabinets. J'ai essentiellement travaillé sur les sujets bancaires et d'assurance. Puis, j'ai passé le troisième concours de l'ÉNA, qui représente $10 \%$ des places et est dédié aux personnes qui viennent du secteur privé ou du secteur associatif. 
Marie Alauzen, Fabien Gélédan, «Un Bercy à l'autre. Entretien avec Maryvonne Le Brignonen, directrice de Tracfin », Revue française d'administration publique, 2021, no2, vol. 178, p. 383-394.

J'avais 32 ans lorsque je suis rentrée à l'ÉNA. Ma scolarité s'est bien passée et je suis sortie «dans la botte » [parmi les premiers du classement de sortie, éligible aux postes ouverts à la Cour des comptes, au Conseil d'État et à l'Inspection générale des finances (IGF)]. À la sortie, je ne souhaitais pas faire du droit, une matière que je n’appréciais pas beaucoup et avais une vision très solitaire du travail au Conseil d'État qui m'a conduite à l'exclure. J'avais envie de sujets à la fois opérationnels, d'actualité, et plutôt tournés vers l'avenir, ce qui m'a conduite à choisir l'IGF, plutôt que la Cour des comptes, puisque l'IGF a une fonction de conseil au gouvernement. J'y ai passé quatre années réglementaires - à l'époque, c'était quatre années indiscutables. J'ai travaillé sur des sujets variés et souvent originaux. J'ai réalisé une mission sur le travail pénitentiaire, c'està-dire la possibilité pour les détenus de travailler dans des ateliers tenus par une régie publique ou par la société privée qui gère la prison dans les établissements qui, en 2010, faisaient l'objet d'un partenariat public-privé. C'était un sujet très original, qui posait de vraies questions de politiques publiques : les prisonniers qui avaient besoin de travailler pouvaient-ils le faire ? Comment trouver une activité qui corresponde à leurs compétences sans concurrencer [de manière déloyale] les entreprises [par le salaire], parce qu'il n'y a pas de salaire minimum en prison? Le travail facilitait-il leur réinsertion? J'ai aussi travaillé sur la maintenance du matériel militaire de la Marine : il y avait à la fois un aspect stratégique et un aspect de programmation des finances publiques. C'était l'époque où l'on s'interrogeait sur l'estimation des coûts de maintenance, d'entretien des grands bâtiments et du matériel de l'armée. L'IGF avait une vraie plus-value à apporter dans le travail réalisé avec des commissaires généraux aux armées. J'ai également participé à des missions un peu plus classiques : à la fin de la Révision générale des politiques publiques (RGPP), dans le domaine du développement durable également dans un domaine plus financier, relatif à l'affacturage, ainsi que sur un rapport relatif à la maîtrise de l'Ondam [Objectif national de réduction des dépenses de l'Assurance maladie]². C'est la grande richesse des thématiques abordées par l'IGF!

À l'issue de mes quatre années, je voulais rester dans le secteur public et m'orienter vers des thématiques régaliennes. Je me suis donc intéressée à la DGFiP, au ministère de l'Intérieur et à la SNCF. La SNCF s'inscrit dans des enjeux de politiques publiques majeurs concernant l'offre de transport, le service à l'usager, l'impact économique ou le fret. J'ai finalement choisi la DGFiP où l'on m'avait proposé deux postes : un poste assez stratégique de chef de bureau, dédié au déploiement du plan stratégique de la direction, et un poste de chef de bureau "les mains dans le cambouis ", à la gestion fiscale des particuliers. Ce second poste se trouvait au cœur du réacteur de la politique fiscale de l'impôt sur le revenu et de ce qui était encore l'impôt sur la fortune. Je voulais être au cœur du réacteur, j’ai donc choisi ce bureau-là. Un an après, le président de la République

${ }^{2}$ Le dernier rapport mentionné est le seul publié sur le site internet de l'IGF : IGF, IGAS, Proposition pour la maîtrise de l'ONDAM (2013-2017), juin 2012, en ligne. 
a décidé la mise en place du prélèvement à la source en France. Il a confié cette mission au Directeur général des finances publiques qui a créé une direction de projet et m'a demandé d'en prendre la tête — poste que j'ai toujours cumulé avec une direction opérationnelle. J'ai d'abord gardé mon poste de chef de bureau, ensuite, j'ai été promu sous-directeur de la gestion fiscale des particuliers et j'ai cumulé les deux postes. J'ai passé cinq années à la DGFiP, cinq années centrées sur le projet de prélèvement à la source. Quand le prélèvement à la source a été lancé, une page se tournait. J'avais l'intention de rester à Bercy. C'est un ministère que j'aime beaucoup, car il anime les politiques publiques en matière économique, budgétaire et fiscale. Après un temps de réflexion, je suis devenu directeur de Tracfin.

Avant d'en venir à Tracfin, pourriez-vous nous expliquer l'activité de l'IGF et le sens de son action?

Il y a deux métiers à l'IGF. Il y a le métier historique, l'inspection. À l'époque où j'y étais - et je crois que cela s'est encore accru - l'inspection était de moins en moins le cœur de l'activité. L'IGF avait muté en un cabinet de conseil du gouvernement. La posture de l'IGF consiste à venir en appui des administrations, leur apporter un toolkit, des réflexions qu'elles n'avaient pas forcément en premier lieu. Il y a d'abord un temps d'écoute, un temps de compréhension des enjeux - même d'ailleurs quand on est en inspection ou en conception de RGPP - et de compréhension du travail quotidien des agents. L'Inspection ne va pas seulement à la rencontre des dirigeants, elle rencontre toutes les catégories d'agents. Cela peut signifier s'asseoir à côté d'un agent d'une catégorie $\mathrm{C}$ pendant une journée, comprendre ce qu'il fait, voir ses problématiques... L'IGF va sur le terrain. Multiplier les expériences de terrain, les entretiens et essayer d'en tirer une substance et des propositions qui vont aider l'administration et le cabinet, c'est la vocation de l'IGF. Notre public, c'était donc les administrations et les cabinets. Pour que certaines politiques publiques ou leur mise en œuvre s'améliorent, il est important qu'il y ait des structures comme l'IGF, l'IGA [nspection générale de l'administration] ou l'IGAS [Inspection générale des affaires sociales] qui ont plus de recul et plus de temps pour aider les administrations, souvent prises par les urgences quotidiennes, à progresser.

Si l'on en vient à votre expérience à la DGFiP, on voit que l'usager y devient une instance beaucoup plus proche. Comment voyiez-vous cette mission? Et qu'est-ce qui change de l'action de l'État que vous serviez de l'IGF à la DGFiP?

Avant même le prélèvement à la source, ce qui m'a beaucoup frappée en arrivant à la DGFiP c'est qu'elle est entièrement tournée vers l'usager. La révolution de l'usager s'était faite une bonne dizaine d'années d'avant. Pour les particuliers, je crois qu'on peut 
Marie Alauzen, Fabien Gélédan, «Un Bercy à l'autre. Entretien avec Maryvonne Le Brignonen, directrice de Tracfin », Revue française d'administration publique, 2021, no2, vol. 178, p. 383-394.

la dater à la mise en place de la télédéclaration, au début des années $2000^{3}$. C'est à cette époque-là que la DGFiP fait sa révolution copernicienne [en référence au programme de déclaration de l'impôt Copernic ${ }^{4}$ ] - à l'époque, la DGI [Direction générale des impôts]. Quand je suis arrivée, en 2014, c'était une direction tournée vers l'usager, avec une volonté de prise en compte de ses problématiques. Le droit fiscal est un droit extrêmement compliqué. Or, la DGFiP a le souci de la manière dont il est mis en œuvre; pour que ce soit la chose la plus simple et la plus compréhensible possible, dans un esprit de service. La Mission relations aux usagers avait été mise en place en 2010. Peu de temps avant que j'arrive en 2014, la Mission de la stratégie des relations au public avait été créée.

Au service de la gestion fiscale, l'usager était en permanence dans nos esprits. Je me souviens des discussions avec la Direction de la législation fiscale qui écrit le droit, un droit désincarné. Nous étions perçus comme des représentants des usagers et comme la direction "poil à gratter", parce qu'on pouvait leur dire que le texte qu'ils avaient construit était certes d'une extrême beauté juridique, mais impossible à mettre en œuvre d'une manière facile et compréhensible pour les usagers, ce qui risquait parfois d'aboutir à une situation surréaliste. J'ai ainsi le souvenir d'une réforme sur la fiscalité des nonrésidents, où, nous étions confrontés à un objet juridiquement complexe, avec beaucoup d'objectifs en termes de droit et de fiscalité, mais qui conduisait à une situation incompréhensible pour l'usager et très difficile à mettre en œuvre.

Quand le prélèvement à la source a été mis en œuvre, c'était non seulement une réforme tournée vers l'usager, mais aussi pour l'usager. Ce n'était pas une réforme de modernisation de l'administration; la DGFiP, dans son volet fiscalité des particuliers, avait déjà mené sa modernisation avec la déclaration de revenus en ligne et la dématérialisation des payements. Ce n'était pas non plus une réforme pour augmenter le taux de recouvrement de l'impôt sur le revenu, il était déjà excellent. C'était une réforme pour les usagers, qui partait du constat simple que payer une année $\mathrm{n}$ un impôt sur ses revenus de l'année n-1, dans un pays où la situation personnelle évolue de plus en plus, était un non-sens. Il y a des cas de chômage, où on peut quitter son emploi salarié pour fonder une entreprise ou l'inverse, il y a des personnes qui partent à la retraite et c'était un non-sens parce qu'on était toujours en décalage. Soit, on ne payait pas assez d'impôts par rapport à ce qu'on gagnait et l'année d'après c'était l'extrême mauvaise surprise parce que les gens qui étaient plus cigales que fourmis se trouvaient en difficulté en dépit d'un salaire confortable. Le cas typique était les jeunes qui commençaient à travailler. Si papa et maman avaient oublié de leur dire : « économise bien tous les mois, tu vas être imposé »,

\footnotetext{
3 Pour une analyse détaillée de ce moment, se reporter à Éric Dagiral, La construction socio-technique de l'administration électronique. Les usagers et les usages de l'administration fiscale, thèse de sociologie, École des ponts et chaussées, 2007.

${ }^{4}$ Pour un examen de Copernic, se reporter à la thèse de Laura Parente, COPERNIC: une étude de l'innovation dans la fiscalité française, thèse de sociologie, École des ponts et chaussées, 2012.
} 
l'année d'après, à la réception de l'avis d'impôt, c'était le drame. A contrario, les personnes qui perdaient leur emploi ou se retrouvaient avec des retraites peu élevées parce qu'elles avaient eu des accidents de vie se trouvaient dans une situation difficile l'année suivante.

L'objectif était - grand terme de l'époque — la « contemporanéisation de l'impôt et du revenu » : l'impôt que je paye tous les mois correspond à ma situation et ceci à deux niveaux. D'abord, il correspond exactement à ce que j'ai gagné, ce que j'ai sur ma fiche de paye ou ce que m'a versé la caisse de retraite, ensuite, il correspond aussi à ma situation, parce que la DGFiP avait mis en place le service « gérer mon prélèvement à la source », une application informatique que nous avons voulue la plus simple, la plus souple possible, où l'usager peut signaler des changements de situation: «je me suis marié», «j'ai divorcé», «j'ai un enfant»... «L’impôt que je paye un mois donné correspond parfaitement à ma situation et l'année après, au mois de septembre, je n'ai quasiment pas de régularisation »; c'est cela l'épure de la réforme.

Nous nous sommes basés sur la réussite de la déclaration de revenus en ligne pour bâtir « gérer mon prélèvement à la source », le système d'information qui sous-tend tout le fonctionnement du prélèvement à la source, où vous pouvez signaler des changements de situation de famille, de revenus, etc. Nous avons également travaillé avec des cabinets de communication sur des tests usagers. Cette pratique est depuis devenue usuelle à la DGFiP : on fait des tests avec des panels représentatifs d'usagers en fonction de leur catégorie socioprofessionnelle, leur origine géographique, etc. Ces tests portaient sur le wording, afin de vérifier si les gens comprenaient ce qu'on leur disait. C'était une nouvelle manière de travailler avec les usagers. Nous avons également organisé tout au début du lancement de la réforme, des [émissions en direct sur le réseau social] Facebook live. Sur la chaîne de la DGFiP, nous organisions tous les jours un live où l'on répondait en direct aux usagers. Je crois que le prélèvement à la source a contribué à adopter des méthodes de communication avec les usagers plus modernes : les webinaires, les chats, etc.

La sensibilité de l'administration fiscale à l'expérience que les usagers font de l'État lors du recouvrement de l'impôt est donc un trait remarquable de la DGFiP, qui a permis le succès de la réforme...

Effectivement, il s'agissait du premier impératif. Le second impératif, en juin 2015, était : «tout doit marcher au 1er janvier 2018 ». Pourtant, la mise en place du prélèvement à la source était un serpent de mer de la réforme de la fiscalité. Il y avait déjà eu un certain nombre de tentatives politiques, qui ne sont jamais allées très loin. Il y avait également beaucoup de rapports, mais ils restaient théoriques et n'avaient jamais réellement envisagé la mise en œuvre de cette réforme. En outre, cette réforme était initialement assez mal accueillie à la DGFiP, parce qu'il y allait avoir un intermédiaire entre nous et le contribuable. Il y avait des résistances externes, mais il y avait aussi des résistances internes à la DGFiP. Lors du premier examen du PLF [projet de loi de finances de l'année] 2016, nous nous sommes rendu compte que ce projet remuait profondément la société française. Il rejouait la question des corps intermédiaires entre l'État et les 
Marie Alauzen, Fabien Gélédan, «Un Bercy à l'autre. Entretien avec Maryvonne Le Brignonen, directrice de Tracfin », Revue française d'administration publique, 2021, no2, vol. 178, p. 383-394.

citoyens. Il y avait des relents d'Ancien Régime, puisqu'on nous a dit à l'Assemblée : «Vous recréez les fermiers généraux!». Ce sujet, qu'on avait cru réglé, ne l'était pas du tout $^{5}$. En conséquence, dans la communication sur la réforme, beaucoup de choses ont été axées sur le fait que le prélèvement à la source restait une relation à l'administration fiscale, donc entre l'État et le citoyen, et que les collecteurs (entreprises, caisses de retraite ou État pour les fonctionnaires) n’étaient que des intermédiaires qui allaient recevoir le minimum d'informations nécessaires et n'allaient faire qu'un calcul très simple. Ils allaient prélever de l'argent pour le remettre le mois suivant à l'État et toutes les questions de déclaration de revenu, de recouvrement du solde, de recouvrement forcé, de changement de situation resteraient entre l'État et le citoyen. C'est vraiment quelque chose qui était très, très fort au départ. Aussi, dans le discours, la communication et la manière dont la réforme a été expliquée en interne, nous avons insisté sur le changement des modalités de recouvrement amiable de l'impôt sur le revenu. Il a été rappelé que cela n'était pas une réforme de l'impôt et ne remettait pas en cause cette relation sacrée entre le contribuable et l'État... En résumé, que nous n'étions pas des apprentis sorciers en train de recréer des corps intermédiaires ni de trahir les acquis de 1789 - parce qu'à un moment, le débat politique et médiatique a commencé à virer comme cela! Ça, c'est la manière dont la réforme a été vue au départ et cela m'avait beaucoup surprise. Je ne pensais pas qu'une réforme plutôt technique éveillerait des craintes aussi lourdes dans la société.

En matière d'organisation interne à la DGFiP, ce qui a été très novateur, c'était l'idée de créer une direction de projet, de gérer le sujet de manière transversale, au-dessus de la mêlée des métiers, et en même temps avec une forte connexion avec les métiers. Cette organisation, alliée à un soutien sans failles de la direction générale, et au fait que nous avons senti une conviction politique forte - les ministres se sont emparés du projet, ont commencé à le soutenir devant la représentation nationale - a permis à la maison DGFiP de comprendre que le projet allait vraiment se faire. Dès que cette prise de conscience a été effective, toute la DGFiP s'est alignée et on a pu vraiment dérouler le projet dans des conditions optimales. Réussir le prélèvement à la source était devenu, je caricature à peine, le projet de chaque agent. Cela nous a animés collectivement. La réputation de l'administration fiscale se jouait. En externe, il y avait la question de ce tiers qui allait intervenir, notamment les entreprises qui n'avaient pas du tout envie de faire le job, parce qu'elles voyaient ça comme une charge complémentaire. Pendant quelques mois a été étudié puis écarté l’idée d’un projet «À la française » : réaliser le prélèvement à la source par les banques, au vu des mouvements sur les comptes. Cela posait un certain nombre de problèmes juridiques et techniques et ce n'était pas un vrai prélèvement à la source. Pour faire un vrai prélèvement à la source, il fallait que les impôts soient prélevés

${ }^{5}$ Les fermiers généraux font partie des références révolutionnaires du débat public en matière de fiscalité. Quelques mois plus tôt, ils s'étaient déjà invités dans les débats sur l'écotaxe. En l'espèce, l'argument a autant été mobilisé par le Front national, que par Attac et les syndicats de la fonction publique, dont Force ouvrière-DGFIP. 
à la source du salaire, donc, par l'employeur. Comment avons-nous fait pour convaincre nos interlocuteurs de devenir de futurs collecteurs? Nous venions en réunion avec des propositions, mais l'objectif était de comprendre les contraintes du collecteur, afin que la mise en œuvre du projet puisse aussi répondre à ses préoccupations. Par exemple, pour les indépendants, les chefs de petites entreprises, le projet visait à ce que tous les mois, ils fassent une mini-déclaration de résultats avec le chiffre d'affaires et les charges pour avoir une base de prélèvement à la source et un taux parfaitement adapté. En discutant avec les fédérations, la CPME (Confédération des petites et moyennes entreprises) et puis l'Union professionnelle des artisans (UPA), nous avons compris combien la solution bâtie dans notre bureau était surréaliste. Cela risquait d'être un enfer pour les chefs d'entreprise et nous sommes arrivés à quelque chose de beaucoup plus pragmatique. Surtout, nos interlocuteurs ont rapidement compris que nous étions ouverts sur les modalités, mais que le point d'arrivée n'était pas négociable. Cela, je le martelais à toutes les réunions.

Ge qui était également intéressant dans cette réforme, c'est le travail interadministration très approfondi, puisque nous nous sommes appuyés sur la DSN, la déclaration sociale nominative, qui relevait de la sphère sociale. Elle devait être prête au

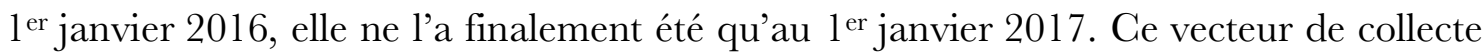
des déclarations sociales permettait d'avoir une déclaration mensuelle numérique de la part des entreprises, ce qui nous permettait de nous insérer dedans sans avoir à déployer un nouveau système de déclarations, de nouvelles charges administratives pour les entreprises, etc.

Le dernier point notable est la collaboration établie avec les éditeurs de logiciels. Nous avons rapidement compris que la petite population d'une quarantaine d'éditeurs de logiciels de paye était l'alpha et l'oméga; si eux n'étaient pas prêts, rien ne serait prêt et la réforme risquait d'échouer. Nous avons décidé de travailler de manière rapprochée avec eux. Nous leur avons proposé de signer une charte avec la DGFiP et ils l'ont accepté. C'était novateur de signer une charte entre une administration et des opérateurs privés. Ils prenaient de leur côté un certain nombre d'engagements sur le respect d'un calendrier, des tests à réaliser pour certaines dates et l'administration s'engageait à prévoir des agents, le temps du projet, pour répondre à leurs questions, pour les aider. Là aussi, il y a eu des intérêts bien compris qui ont permis que tout le monde soit in fine arrivé à bon port, à la bonne date. En outre, le report d'un an de la réforme a certainement été bénéfique pour qu'elle soit plus finement ajustée, par exemple concernant l'avance de crédit d'impôt au début de chaque année, et a permis un important travail de pédagogie et de conviction.

Dans ces ajustements, comment avez-vous pris en considération ce que l'on appelle classiquement la "fracture numérique», ici le fait que tout le monde ne soit pas forcément équipé pour aller régulièrement ajuster son taux de prélèvement?

Elle a été prise en compte de deux manières. La DGFiP s'était engagée à avoir des centres d'assistance téléphonique, développés et généralisés à l'occasion du 
Marie Alauzen, Fabien Gélédan, «Un Bercy à l'autre. Entretien avec Maryvonne Le Brignonen, directrice de Tracfin », Revue française d'administration publique, 2021, no2, vol. 178, p. 383-394.

prélèvement à la source. Les personnes qui n'étaient pas à l'aise avec le numérique pouvaient, au téléphone, faire faire les opérations nécessaires à la mise en place du prélèvement à la source. Avoir ce numéro gratuit pour faire ces démarches a été une manière de traiter la fracture numérique. C'était un premier point. Second point: la généralisation de bornes numériques dans les centres des finances publiques, auxquelles les usagers accèdent avec présence d'agents ou d'alternants, qui les guident dans leurs démarches.

L'une des raisons pour lesquelles nous nous interrogions sur la fracture numérique c'est parce que c'est une des formes de résistance les plus visibles, une occasion de dire : "cet État qui me ponctionne sans que je le voie, je n'en veux pas ». Nous nous demandions s'il y en avait eu d'autres ou comment elles avaient été formulées.

Je pense que nous avons répondu de deux manières à la question de «l'État me ponctionne sans que je le voie, je n'en veux pas ». Il était important que les gens, dans les premiers mois qui ont suivi la réforme, comprennent leur nouveau salaire net et saisissent la différence entre celui-ci et leur ancien salaire net. Ce qu'avaient prévu les textes a été mis en œuvre par les éditeurs de logiciels de manière très précise : les usagers voyaient le net à payer avant prélèvement à la source et le net à payer après le prélèvement à la source. Nous partagions un idéal de transparence : que chacun puisse mesurer chaque mois combien l'État lui a pris. C'était le premier point. Le deuxième point était l'un des axes de communication que nous avons beaucoup utilisé avant la réforme : le prélèvement à la source permet aux gens de devenir acteurs de leur fiscalité, de l'impôt qu'ils payent. Au lieu de se voir prélever des tiers provisionnels calculés sur des revenus anciens et d'avoir une régularisation au mois de septembre, ils peuvent ajuster tous les mois l'impôt à leur situation. Ils ont la main pour le faire, signaler des changements de situation de famille: des mariages, des naissances, des décès ou des évolutions de situation professionnelle. L'un des axes de communication était de montrer aux gens qu'ils pouvaient avoir beaucoup plus la main qu'auparavant, même si ça passait par une interface numérique et par un tiers collecteur.

Pourriez-vous revenir sur l'idée de rendre le contribuable actif? N'y a-t-il pas eu de réticences de l'intérieur de la DGFiP, sur les fraudeurs ou de ceux qui allaient utiliser cela à des fins plus ou moins honnêtes ou à leur avantage pour prendre connaissance des mécanismes de calcul fiscal et déjouer une partie du contrôle opéré par l'État?

Cela a suscité un certain nombre de questions, mais il ne faut pas oublier que l'année d'après il y a toujours la déclaration de revenus. La déclaration de revenus remet les pendules à l'heure et rattrape ceux qui auraient éventuellement « fraudé », sachant que tant que la déclaration de revenus n'est pas faite, on est dans un décalage de trésorerie, pas dans une fraude. C'est toutefois un point qui a été soulevé. De plus, il ne faut pas oublier que le prélèvement à la source a été déployé en même temps que les dispositifs d'échange d'information entre les États membres de l'Union européenne et les États de 
l'OCDE, qui permettent de mieux identifier les comptes et les revenus à l'étranger. Avec le recul, on constate qu'il y a eu beaucoup plus de résistances philosophiques et politiques à la création d'un intermédiaire entre l'administration et le citoyen, que sur les questions de la fraude.

Avez-vous observé des résurgences ou des effets concrets des problèmes qui venaient de cette intermédiation de l'entreprise dans la relation entre l'usager et les services fiscaux de l'État?

Je ne sais pas s'il y a eu des résurgences. À l'époque, les problématiques portaient sur la connaissance que l'entreprise pouvait avoir de la situation financière et personnelle de ses salariés. On le sait, en France, l'argent est un sujet un peu sale, tabou. Certains craignaient que l'entreprise puisse connaître les revenus de votre conjoint, ou votre patrimoine, au travers des réductions d'impôt. Notre réponse était que la seule chose transmise à l'entreprise était le taux du prélèvement à la source. Nous avons démontré au cours de très nombreuses réunions d'élus, de chefs d'entreprise, que derrière un taux de prélèvement à la source il pouvait y avoir des situations multiples, et par conséquent, l'employeur ne pourrait pas en tirer de conclusions. Ces critiques, très fortes au début du projet, ont rapidement décru. Une autre crainte des entreprises - qui je crois ne s'est pas vérifiée, mais il faudrait en parler avec elles - , était que les salariés, négocient leur salaire net après prélèvement à la source et donc introduisent une distorsion dans la discussion puisque le taux de prélèvement à la source est individualisé et qu'à rémunération égale vous pouvez avoir des taux de prélèvement très différents. Je ne crois pas que cette crainte se soit confirmée, je n'ai rien lu dans la littérature depuis qui l’indique.

Passons à votre expérience à Tracfin. Vous avez quitté une mission qui vous avait mise au contact direct du contribuable, des usagers et aussi des agents opérationnels. Avec Tracfin, vous accèderez à une tout autre expérience du service de l'État. Pourriez-vous nous dire pourquoi vous avez choisi de vous diriger vers ces sujets, loin de ce sur quoi vous avez travaillé, du moins en apparence?

Les carrières administratives sont faites de surprises et de changements de direction. Venir à Tracfin n'était pas sur ma feuille de route. Je pensais rester à la DGFiP, y faire d'autres métiers, travailler sur d'autres thématiques comme le contrôle fiscal ou la relation aux administrations territoriales. Les hasards, les rencontres, les opportunités ont mené Tracfin sur ma route : ça restait dans la sphère Bercy, au moins sur la partie fraude, ça parlait de choses que j'avais pu connaître à la DGFiP. Puis, la lutte contre le financement du terrorisme et la défense des intérêts fondamentaux de l'État me paraissaient purement régaliennes. C'est aussi une mission de politiques publiques pour l'usager, pour protéger sa sécurité même si c'est quelque chose de lointain, de transparent, qui peut faire peur. Voilà comment je suis arrivée à Tracfin. Alors est-ce que c'est une figure de l'État différente? Oui, puisque c'est l'État de l'ombre, l'État que personne ne connaît, dont on voit les effets de l'action uniquement quand cela ne marche pas ou quand c'est mal fait. Par exemple, lors des scandales de blanchiment ou de fraude, et des opérations terroristes qui réussissent. À la DGFiP, quand vous ratez une campagne 
Marie Alauzen, Fabien Gélédan, «Un Bercy à l'autre. Entretien avec Maryvonne Le Brignonen, directrice de Tracfin », Revue française d'administration publique, 2021, no2, vol. 178, p. 383-394.

d'impôt sur le revenu cela se voit, mais quand cela réussit cela se voit aussi. Alors qu'à Tracfin, quand nous faisons bien notre travail, le citoyen a l'impression qu'il ne se passe rien. C'est très différent dans la manière dont on peut se positionner en tant que fonctionnaire et cela peut être frustrant. Quand on voit les suites judiciaires données aux dossiers, quand on voit qu'il y a une modification de la pratique du ministère du Travail sur le dispositif de chômage partiel pour limiter les fraudes, qu'en matière de lutte contre le financement du terrorisme, il y a des opérations menées par le Parquet national antiterroriste, nous produisons une communication maîtrisée de l'action publique, avec une mise en lumière limitée au strict nécessaire. À Tracfin, c'est l'État très profond, pas dans le sens qu'utilisent les Américains, mais l'État caché. C'est une action souterraine. Pour les citoyens, Tracfin reste assez mystérieux. Les citoyens ne se rendent pas compte de ce qui est lié à notre politique publique. Quand vous ouvrez un compte bancaire ou une assurance vie, les questionnaires que requièrent les banques ou les assureurs, qui demandent d'où vient et où va aller l'argent ont un rôle dans la lutte contre le blanchiment et le financement du terrorisme, mais les citoyens n'en ont souvent pas conscience.

En vous écoutant, on peut se demander si, dans cet État de l'ombre, vous n'avez pas un rapport un peu différent, au-delà des citoyens, avec le politique ou avec d'autres corporations. Vous avez mentionné le rôle de certaines corporations partenaires de la DGFiP, comme les éditeurs de logiciels. À la lecture du rapport d'activité de Tracfin', on trouve une liste de professionnels qui font partie de la chaîne de l'information, indispensable au travail du service. Comment l'État se présente-t-il à travers vous pour ces professionnels?

Ces professionnels sont assujettis par la Loi à la lutte contre le blanchiment et le financement du terrorisme $[\mathrm{LC} / \mathrm{BFT}]^{7}$. Ils doivent, sous peine de sanctions, mettre en œuvre un certain nombre de diligences de lutte contre le blanchiment et faire des déclarations de soupçon à Tracfin, à partir du moment où ils savent, soupçonnent, ont de bonnes raisons de soupçonner, une opération de blanchiment, de fraude ou de financement du terrorisme. Le droit est très coercitif: tout est placé sous l'angle de l'obligation et de la sanction. Cela n'empêche pas que la société évolue, elle est en quête de sens et nos assujettis, en tant que personnes morales, sont aussi en quête de sens. Ils ont besoin de savoir pourquoi ils nous transmettent l'information. Il n'y a aucune contestation de leur part du fait qu'ils doivent nous transmettre cette information. En revanche, ils veulent savoir à quoi cela servira. La ligne rouge est que, sur un dossier particulier, ils ne sauront jamais, précisément, comment nous avons utilisé leur information. Ils sont déliés de leurs obligations et responsabilités pénales dès lors qu'ils transmettent une déclaration

6 Tracfin, Rapport annuel d'activité 2019, ministère de l'Économie, des Finances et de la Relance, disponible en ligne.

${ }^{7}$ Les personnes assujetties aux obligations de lutte contre le blanchiment des capitaux et le financement du terrorisme sont listées aux articles L561-2 à L561-4 du Code monétaire et financier. 
de soupçon, et nous leur devons un retour sur ce pour quoi ces déclarations sont utilisées : le type de fraudes, de blanchiment, de financement du terrorisme qu'on identifie. Nous devons aussi leur donner ces informations en rétroaction pour améliorer la qualité des déclarations de soupçon. Nous pouvons encore progresser là-dessus. La modernisation de la relation aux assujettis est quelque chose que je mets sur mon plan de travail pour 2021. Cela passe aussi, dans une société numérique, par une modernisation de l'interface informatique sur laquelle les assujettis envoient les déclarations de soupçon. Je pense que nous pouvons progresser encore en termes de pédagogie, d'explicitation, pour faciliter la saisie de la déclaration de soupçon. Finalement, d'autres administrations tournées vers le public et dans la lumière, comme la DGFiP, on a aussi ces problématiques de donner du sens, de faire de la pédagogie, de convaincre et d'offrir un meilleur service. Il faut approfondir cette piste.

Et sur le volet de la relation avec le politique, peut-être y a-t-il une différence avec la DGFiP ou l'IGF?

Tracfin a une double identité. C'est à la fois la cellule de renseignement financier française au sens du GAFI [Groupe d'action financière], et tant les standards du GAFI que la législation européenne prévoient que les cellules de renseignement financier sont «opérationnellement autonomes ». Cela signifie que ce sont des services bien identifiés dotés d'un budget propre, qui ne prennent pas d'instructions quand ils traitent et transmettent des informations. C'est très clair, il y a une étanchéité forte vis-à-vis du politique. Tracfin est aussi un service de renseignement du premier cercle. Or, les services de renseignement sont là pour protéger les intérêts supérieurs de la nation érigés par le pouvoir politique. La lutte contre le terrorisme est une politique publique de premier ordre, donc la lutte contre le financement du terrorisme, qui est une des missions de Tracfin en matière de défense des intérêts fondamentaux de la nation, est une politique de premier ordre, que nous traitons en priorité. Il en va de même pour la prédation économique et financière des entreprises innovantes, sujet de préoccupation pour les ministères économiques et financiers.

Tracfin a également joué un rôle dans le cadre de la mise en place des dispositifs de chômage partiel liés à l'arrivée de l'épidémie de Covid-19. Pouvez-vous nous dire comment cela s'est passé dans un contexte d'urgence et d'incertitudes?

Au moment du premier confinement et de la mise en place massive du chômage partiel, le politique voulait avoir des dispositifs d'aide aux entreprises immédiatement opérationnels. L'objectif était très clair : il fallait que l'argent puisse être versé très rapidement. Ce qui implique des contrôles a priori limités et plutôt des contrôles a posteriori. Tracfin ne travaille pas d'initiative et cette alerte a émané des signalements envoyés par les professionnels assujettis, notamment des établissements de crédit, qui laissaient soupçonner des fraudes au chômage partiel. Nous avons fait nos diligences classiques pour contextualiser ces soupçons, en ayant accès à la fois aux éléments financiers, le cas échéant 
Marie Alauzen, Fabien Gélédan, «Un Bercy à l'autre. Entretien avec Maryvonne Le Brignonen, directrice de Tracfin », Revue française d'administration publique, 2021, no2, vol. 178, p. 383-394.

aux éléments comptables, aux bases de l'administration fiscale et de l'administration sociale. Nos enquêtes ont permis d'étayer un soupçon de fraude au dispositif de chômage partiel et de la reprise d'entreprises en difficulté. Après avoir informé nos ministres de tutelles, ces typologies de fraude détectées ont permis de mener des travaux conjoints, notamment avec la Mission interministérielle de coordination antifraude (MICAF) et le ministère du Travail, puis d'alerter rapidement nos instances gouvernementales. C'est assez topique de la manière dont Tracfin travaille et peut avoir une action interministérielle.

Comment faites-vous pour incarner à la fois la discrétion du service de renseignement et les apparitions publiques où vous êtes à ce moment-là la tête de l'État pour des usagers très particuliers?

La césure est très nette. La ligne rouge est la même pour moi et pour les cadres du Service qui peuvent être amenés à s'exprimer à l'extérieur : on ne parle, hors de Tracfin et hors du cercle du renseignement, jamais d'opérationnel, jamais de dossier, jamais d'un dossier particulier. Quand je dois incarner Tracfin à l'extérieur, à la rencontre des professionnels, dans des apparitions publiques, dans le monde étudiant ou vis-à-vis de médias extrêmement choisis et sélectionnés, je vais évoquer des typologies de fraude, de blanchiment, de financement du terrorisme pérennes ou innovantes et notre actualité institutionnelle. C'est ainsi que nous avons communiqué rapidement à nos assujettis sur les modes de fraudes au plan de relance. À Tracfin, nous avons une cellule d'analyse stratégique. Le rôle de cette cellule est d'identifier les tendances de blanchiment de capitaux, de fraudes ou de financement du terrorisme par l'analyse des signalements reçus, pour en tirer une substantifique moelle, des typologies, des problématiques. C'est la seule matière dont on va parler à l'extérieur. C'est la ligne rouge absolue. Dans les médias, nous déclinons toujours des sollicitations qui amèneraient à parler d'un dossier spécifique, car lorsque Tracfin externalise un dossier à ses partenaires (service de renseignement, autorité judiciaire ou autres partenaires), une publicité précoce peut nuire aux investigations en cours. D'autant plus que l'établissement des déclarations de soupçon est couvert par une stricte confidentialité. Tracfin place le secret des déclarations et la protection du déclarant au cœur du dispositif lutte contre le blanchiment d'argent et le financement du terrorisme. Nous nous interrogeons toujours pour savoir si notre communication sera utile - aux professionnels assujettis, au grand public - tout en étant suffisamment habillée et maquillée pour ne pas trahir des dossiers en cours. C'est une parole plus parcimonieuse que celle de l'administration fiscale, même si dans l'administration fiscale, il y a aussi la question du secret fiscal. En conclusion, nous examinons de manière très précise l'opportunité de s'exprimer. 